\title{
Influence of amorphous layers on the thermal conductivity of phononic crystals
}

\author{
Maxime Verdier and David Lacroix ${ }^{*}$ \\ Université de Lorraine, CNRS, LEMTA, F-54000 Nancy, France \\ Stanislav Didenko, Jean-François Robillard, ${ }^{\dagger}$ and Evelyne Lampin ${ }^{\ddagger}$ \\ Université de Lille, CNRS, Centrale Lille, ISEN, Université de Valenciennes, UMR 8520, IEMN, F-59000 Lille, France \\ Thierno-Moussa Bah \\ STMicroelectronics, 38920 Crolles, France \\ and Université de Lille, CNRS, Centrale Lille, ISEN, Université de Valenciennes, UMR 8520, IEMN, F-59000 Lille, France \\ Konstantinos Termentzidis ${ }^{\S}$ \\ Univ. Lyon, CNRS, INSA-Lyon, Université Claude Bernard Lyon 1, CETHIL UMR 5008, F-69621 Villeurbanne, France \\ and Université de Lorraine, CNRS, LEMTA, F-54000 Nancy, France
}

(Received 8 December 2017; published 21 March 2018)

\begin{abstract}
The impact of amorphous phases around the holes and at the upper and lower free surfaces on thermal transport in silicon phononic membranes is studied. By means of molecular dynamics and Monte Carlo simulations, we explore the impact of the amorphous phase (oxidation and amorphous silicon), surfaces roughness, and a series of geometric parameters on thermal transport. We show that the crystalline phase drives the phenomena; the two main parameters are (i) the crystalline fraction between two holes and (ii) the crystalline thickness of the membranes. We reveal the hierarchical impact of nanostructurations on the thermal conductivity, namely, from the most resistive to the less resistive: the creation of holes, the amorphous phase around them, and the amorphization of the membranes edges. The surfaces or interfaces perpendicular to the heat flow hinder the thermal conductivity to a much greater extent than those parallel to the heat flow.
\end{abstract}

DOI: 10.1103/PhysRevB.97.115435

\section{INTRODUCTION}

With the development of new nanofabrication techniques, new classes of materials with particular physical properties have emerged. For example, ultrathin membranes with or without holes or pillars are interesting candidates to tune heat transport. Among them, the so-called phononic crystals $(\mathrm{PnC})$ are structures with periodic hole patterning, which have attracted scientific interest these past years due to fundamental questions in physics but also for their potential applications in thermal management. The characteristic sizes of this class of materials are now of the order of some tens of nanometers, enabling the modification of thermal phonon propagation in the terahertz regime [1]. Geometric parameters such as the hole diameter, their period, and their ordering as well as the coherent or incoherent nature of phonon transport within the membranes were the target issues of several experimental and theoretical studies [1-7]. In general, important reduction of the thermal conductivity (TC) in nanoscale PnC was observed, reaching the amorphous limit in several cases [8]. Nevertheless, there are debates about significant discrepancies between measure-

\footnotetext{
*david.lacroix@univ-lorraine.fr

$\dagger$ jean-francois.robillard@isen.iemn.univ-lille1.fr

$\ddagger$ evelyne.lampin@isen.iemn.univ-lille1.fr

${ }^{\S}$ konstantinos.termentzidis@insa-lyon.fr
}

ments and simulations or models $[9,10]$ and the temperature dependence of coherent boundary scattering [10,11].

Several developments have been made in past years in this research field. Wagner et al. [1] found that the coherent acoustic phonon modes are suppressed with the introduction of disorder in hole alignment. Nakagawa et al. [12] showed that hole spacing has a strong influence on TC when the period is within the range of the thermal phonon mean free path. Incoherent boundary scattering depends only on the shape, size, and separation of the holes, while coherent boundary scattering additionally depends on symmetries according to how these holes are distributed [10]. Dechaumpai and Chen [13] highlighted the importance of the zone folding effect on thermal transport in PnCs. Lee et al. [14] investigated the transition from ballistic to diffusive regimes in holey silicon, stretching the importance of the long-wavelength phonons, longer than the characteristic geometries (neck size, thickness of membranes), for heat transport. A very recent experimental study by Nomura et al. [15] on the thermal properties of crystalline silicon and amorphous $\mathrm{SiGe} \mathrm{PnCs}$ with the same geometric parameters showed a relative reduction of the TC in the two structures by a factor of 20. Hopkins et al. [3] measured the cross-plane TC of a crystalline silicon $\mathrm{PnC}$ and found a very low value $\left(6.8 \mathrm{~W} \mathrm{~m}^{-1} \mathrm{~K}^{-1}\right)$. In the latter study the measured TC was much smaller than the predicted one when only accounting for boundary scattering at the interfaces of the PnC. Thus, the authors assumed that this phenomenon is related to the 
coherent phononic effects, which are causing an additional reduction to the cross-plane TC, but they never considered oxidation or amorphization as the cause of the strong reduction. In addition, it has been recently shown that coherence effects in silicon PnCs can occur when the temperature is very low $(T<20 \mathrm{~K})$ [16]. At room temperature, the reduction of the thermal conductivity as compared to the bulk is mostly due to porosity, boundary scattering, and amorphization, oxidation, and/or roughness of the structure. The relative impact of these parameters is not fully understood. Here we study the hierarchical impact of the interfaces, regarding their nature and their orientation compared to the heat flux propagation direction.

Concerning the roughness or the existence of amorphous shells in etched nanostructures, the existing literature discusses some advances but there are still several issues to be quantified and studied. Yanagisawa et al. [17] suggested very recently that the TC depends less on the period of the PnC nanostructures than on the surface roughness. They also claim that the smallest length of the structure (the neck) is the most important parameter in TC reduction. One can imagine that the picture is even more complex when there are interactions between vibrations of amorphous and crystalline materials at the interfaces between the two phases. There is indeed an impact of the amorphous phase in the vicinity of the crystalline phase and thermal transport through amorphous/crystalline interfaces is a subject under investigation in the nanoscale heat transfer community [18]. Damart et al. [19] showed that the amorphous/crystalline interfaces act as a low-energy pass filter slowing down the high kinetic energy motion for all spectra of vibration frequencies. A couple of studies on crystalline/amorphous superlattices $[20,21]$ revealed interesting findings for the minimum distance from the interfaces for the phonons to retrieve the bulk density of states. Neogi et al. [22] in a recent article showed the major impact of the surface nanostructuration and the amorphous edges of membranes on the TC. By means of molecular dynamics simulations they calculated that the amorphization and nanostructuration of 8-nm-thick membranes can reduce the TC by a factor of 10 . Another work demonstrated that amorphous shells [23] around spherical nanopores can drastically reduce thermal transport properties. Here we investigate the impact on TC of the thickness of the surface native oxide and the amorphous shells around the nanoholes. This subject has never been treated in $\mathrm{PnC}$ nanostructures and it can bridge the discrepancy observed between measurements and theoretical calculations [9].

In order to illustrate the relevance of our modeled structures, we present a state-of-the-art fabrication process of PnCs in which amorphization is clearly observed. Crystalline silicon membranes of thicknesses down to $50 \mathrm{~nm}$ and surface area of $150 \mu \mathrm{m} \times 20 \mu \mathrm{m}$ are fabricated and suspended [Fig. 1(a)]. The membrane is perforated according to a square lattice of periodic holes as can be seen in Fig. 1(a). The samples are prepared by means of electron beam lithography and reactive ion etching. In order to obtain submillimeter size arrays of 35- to 50-nm-diameter holes in a limited time (a few hours), we used the so-called dots-on-the-fly methodology $[24,25]$. The minimum period $(60 \mathrm{~nm})$ at a hole diameter of $35-40 \mathrm{~nm}$ is limited by the occurrence of proximity effects [26].
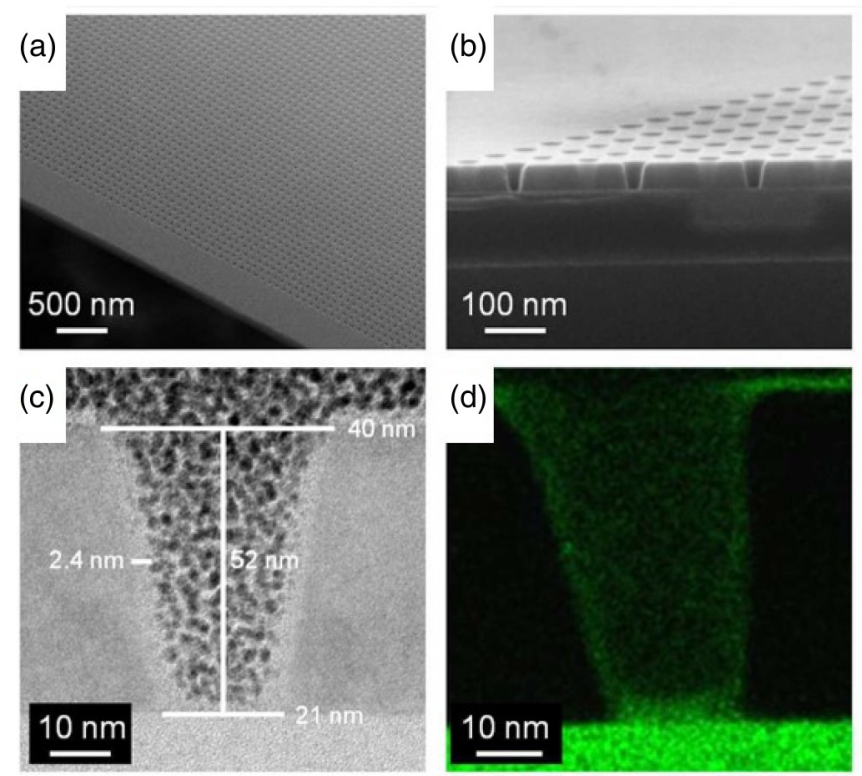

FIG. 1. (a) SEM picture of a 59-nm-thick nanostructured silicon membrane. (b) SEM cross-section view of a nanostructured siliconon-insulator layer before membrane release. The stack is composed of $55 \mathrm{~nm} \mathrm{Si}$ over $145 \mathrm{~nm}$ buried oxide $\left(\mathrm{SiO}_{2}\right)$. (c) Scanning transmission electron microscope (STEM) picture of a partially etched hole. (d) Electron dispersive x-ray spectroscopy (EDX) analysis showing silicon (black) and oxygen (green) content.

A cross-section scanning electron microscopy (SEM) image is shown in Fig. 1(b), which exhibits the perforated silicon layer, the BOX layer, and the handler wafer. To investigate the quality of the holes edge, samples were analyzed by means of transmission electron microscopy (TEM). Figure 1(c) presents a cross-section image of a single hole. In this image it can be seen that the actual silicon thickness is $52 \mathrm{~nm}$ and a thin 2- to 3 -nm layer is present on the membrane upper side as well as on the hole surface. The nature of this layer is confirmed to be at least partially oxide by the electron dispersive $\mathrm{x}$-ray analysis in Fig. 1(d), in which the oxygen content is highlighted. It can be argued that amorphous silicon or silicon oxide can be found at the hole edges and at the membrane surfaces, natively or after exposure to air [22]. Thus, modeling $\mathrm{PnC}$ with amorphous shells around holes and amorphous edges at the top and the bottom of the membrane is of great interest and more realistic when compared to the elaborated structures.

The paper is organized as follows: after this introduction the modeling of structures is described and the molecular dynamics (MD) and the Monte Carlo (MC) methodologies to calculate the thermal conductivity are explained. In Sec. III, the effect on thermal conductivity of the amorphization of holes with amorphous silicon and amorphous silicon dioxide is presented. Many parameters are studied, such as the amorphous phase thickness, the hole diameter, and the period of the hole pattern. In the same section, membranes with amorphous edges with and without periodic cylindrical holes are studied. Eventually, MC simulations are conducted with a roughness model to assess the importance of surface specularity in measurements and modeling of the thermal properties of nanostructures. In the last section, conclusions and discussions are given. 
TABLE I. Characteristic sizes of elaborated and MD simulated PnC membranes. For the geometric parameters mentioned here, see Figs. 2 and 6.

\begin{tabular}{lccc}
\hline \hline Parameter & MD membranes & Elaborated membranes & MD/Expt. ratio \\
\hline Membrane thickness, $h$ & $15 a_{0}=8.145 \mathrm{~nm}$ & $60 \mathrm{~nm}$ & 0.136 \\
Pitch size (period), $a$ & $15 a_{0}=8.145 \mathrm{~nm}$ & $60 \mathrm{~nm}$ & 0.136 \\
Hole diameter, $d$ & $10 a_{0}=5.43 \mathrm{~nm}$ & $21-50 \mathrm{~nm}$ & $0.258-0.109$ \\
Hole silica/a-Si shell thickness $t_{s}$ & $(1-2) a_{0}=0.5-1 \mathrm{~nm}$ & $2-3 \mathrm{~nm}$ & $0.16-0.5$ \\
Porosity $\phi$ & $34.9 \%$ & $34.9-38.5 \%$ & 1 \\
Neck size & $(3-13) a_{0}=1.6-7 \mathrm{~nm}$ & $17-36 \mathrm{~nm}$ & $0.1-0.2$ \\
\hline \hline
\end{tabular}

\section{SIMULATION METHODS AND MODELING}

\section{A. Structure modeling}

Several systems are modeled with MD to investigate the impact of amorphous shells around the holes and amorphous edges at top and bottom surfaces of the membrane on the thermal conductivity. The shell contains either amorphous silicon (a-Si) and/or silica $\left(\mathrm{a}-\mathrm{SiO}_{2}\right)$. On the one hand, the ratio of hole diameter to hole period in elaborated membranes presented in the Introduction is preserved in MD simulations downsizing the system by a factor close to 7; on the other hand, the same factor is applied to reduce the membrane thickness while preserving the porosity. This choice has been made due to computational resource limitations related to the use of the equilibrium molecular dynamics method (EMD). In Table I the characteristic sizes of elaborated and MD modeled membranes are given. The thicknesses of membranes in this section are kept constant and equal to $h=15 a_{0}(8.145 \mathrm{~nm})$ as well as the diameter of the holes, $d=10 a_{0}=5.43 \mathrm{~nm}$ (where $a_{0}=5.43 \AA$ is the cubic lattice constant of crystalline silicon). We consider periodic cylindrical nanoholes organized in a square lattice in silicon membranes, with or without a-Si or $\mathrm{a}_{-} \mathrm{SiO}_{2}$ shells around them (Fig. 2).

Four distinct cases are studied: crystalline silicon PnCs without shell around the holes [Fig. 2(a)], c-Si PnC with a-Si

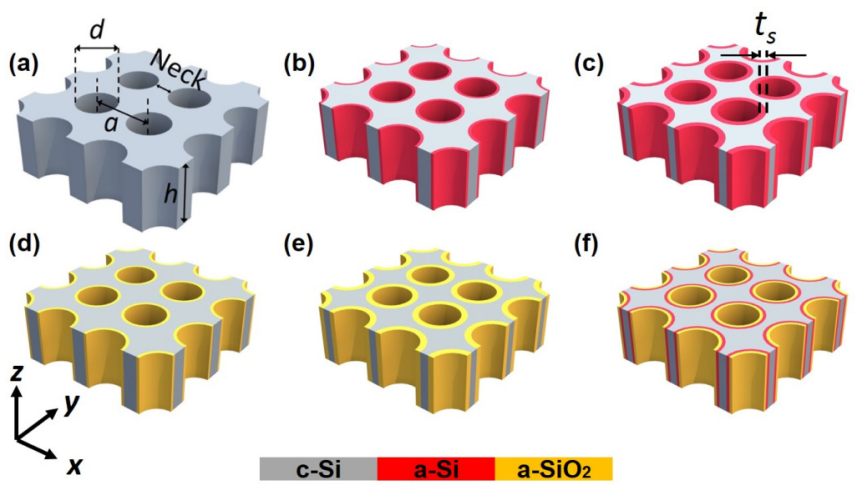

FIG. 2. Systems modeled with MD to investigate the impact of the nature of the hole shell on TC. (a) Crystalline silicon without shell, (b) amorphous silicon shell with thickness $0.5 \mathrm{~nm}$, (c) amorphous silicon shell with thickness $1.0 \mathrm{~nm}$, (d) amorphous a- $\mathrm{SiO}_{2}$ shell with thickness $0.5 \mathrm{~nm}$, (e) amorphous a- $\mathrm{SiO}_{2}$ shell with thickness $1.0 \mathrm{~nm}$, and (f) double shell with a- $\mathrm{SiO}_{2}$ and a-Si, each with thickness $0.5 \mathrm{~nm}$. $d$ is the pore diameter, $a$ is the period of the lattice, $h$ is the membrane height, $t_{s}$ is the shell thickness, and the neck is the distance between the edges of two neighboring pores. shell with shell thicknesses $t_{s}$ of 0.5 and $1.0 \mathrm{~nm}$ [Figs. 2(b) and 2(c)], c-Si PnC with amorphous a-SiO 2 shell with $t_{s}=0.5$ and $1.0 \mathrm{~nm}$ [Figs. 2(d) and 2(e)], and c-Si PnC with a double shell of a-SiO 2 and a-Si with a total $t_{s}=1 \mathrm{~nm}$ [Fig. 2(f)]. The axes of the cylindrical holes are along the $z$ axis. Periodic boundary conditions are set in the $x$ and $y$ directions, while in the $z$ direction fixed boundary conditions are used to model the upper and lower surfaces of the membrane. For the dimensions studied in Sec. III A, all these systems contain between $1.6 \times 10^{4}$ and $1.9 \times 10^{4}$ atoms.

To model in a systematic way nanostructures containing both crystalline and amorphous phases a very specific procedure was applied to ensure the reliability of nanostructure design [27]. In this procedure, called the "cut and paste technique," we start from the bulk amorphous phase (a-Si or $\mathrm{a}-\mathrm{SiO}_{2}$ ). Then some atoms in specific regions of the latter bulk are deleted and replaced by atoms with positions corresponding to a bulk crystal system in order to obtain an amorphous cylinder inside a crystalline matrix. Finally, atoms of amorphous and crystalline phases which are too close to each other are removed, and the hole is created by deleting amorphous atoms at the center of the cylinder. We have to stress here the importance of modeling nanostructures containing both crystalline and amorphous phases and the precautions one should take into account, such as a good radial distribution function of the amorphous phase, the constant atomic energies inside crystalline and amorphous phases, and the interfacial energy between the two phases which correspond to the experimental one [27]. The method has been applied with success for different nanostructured materials such as superlattices [20], nanowires [28,29], and nanoporous structures [23].

\section{B. Equilibrium molecular dynamics}

Molecular dynamics simulations are performed with LAMMPS [30]. The TC is determined using EMD method thanks to the Green-Kubo formula [31]:

$$
\kappa_{\alpha \beta}=\frac{\mathcal{V}}{k_{B} T^{2}} \int_{0}^{+\infty}\left\langle J_{\alpha}(t) J_{\beta}(0)\right\rangle d t,
$$

where subscripts $\alpha$ and $\beta$ denote directions, $\mathcal{V}$ is the system volume, $J$ is the heat flux, $t$ is the time, $T$ is the system temperature, and $k_{B}$ is the Boltzmann constant. With EMD, the TC can be obtained for all directions. $J$ is calculated as

$$
J=\frac{1}{\mathcal{V}}\left[\sum_{i}\left(E_{k, i}+E_{p, i}\right) v_{i}-\sum_{i} \sigma_{i} v_{i}\right],
$$


where $E_{k}$ and $E_{p}$ are the kinetic and potential energies, respectively, $\sigma$ is the stress tensor, $v$ is the velocity, and the sum is made over all atoms. The time step is always set to $0.5 \mathrm{fs}$. First, the system is relaxed at $T=300 \mathrm{~K}$ using an NVT ensemble with a Nose-Hoover thermostat for 200 ps. After this equilibration step, no artificial modes due to the initial conditions are observed (ringing modes [32,33]). Then the computation of the TC starts, evaluating the flux fluctuation correlation with a time window of $40 \mathrm{ps}$, for a total duration of $10 \mathrm{~ns}$. In order to reduce the uncertainty of the results, the TC is time averaged over the last $5 \mathrm{~ns}$ of each simulation and ensemble averaged for ten different initial configurations. Periodic boundary conditions are applied at surfaces perpendicular to the $x$ and $y$ directions, while fixed conditions are applied at free surfaces (perpendicular to the $z$ direction for plain membranes and $\mathrm{PnCs}$ ).

For systems containing only silicon atoms, their interactions are described with the Stillinger-Weber potential [34] with the modified coefficients proposed by Vink et al. [35] as this parametrization is able to accurately describe thermal transport in silicon, for both crystalline and amorphous phases. When the system contains oxygen (in $\mathrm{a}_{-} \mathrm{SiO}_{2}$ shells), the Tersoff $\mathrm{SiO}$ [36] interatomic potential is used instead of the Stillinger-Weber potential. To conclude on this part, it is noted that the TC for bulk Si using the Stillinger-Weber potential is $\kappa=164 \pm 21 \mathrm{~W} \mathrm{~m}^{-1} \mathrm{~K}^{-1}$. That is in good agreement with experimental values $[37,38]$. The consistency of our study has been checked by computing the TC of one of our systems with both potentials (Stillinger-Weber and Tersoff) and the results are reasonably in agreement (difference less than 5\%). It was also checked that the thermal conductivity obtained by EMD was the same when we use larger simulation cells (containing four holes).

\section{Monte Carlo for phonons}

Heat transport at the nanoscale can be described with the Boltzmann transport equation (BTE). In the relaxation-time approximation, this equation becomes $[39,40]$

$$
\frac{\partial f}{\partial t}+\vec{v}_{g} \cdot \vec{\nabla} f=\frac{f^{0}-f}{\tau(\omega, T)},
$$

where $f$ is the phonon distribution function, $v_{g}$ is the group velocity, $\tau$ is the relaxation time, and the superscript " 0 " denotes thermal equilibrium.

The Monte Carlo method resolves this equation, considering phonons as moving particles with spectral properties like frequency, polarization, and velocity. Phonon populations are initialized according to the phonon density of states (DOS) of the material, following a Bose-Einstein distribution. A temperature gradient is imposed by regularly reinitializing phonon populations at both ends of the system. At each time step, after its displacement, a phonon has a chance to experience impurity, normal, or umklapp scattering. The probability of each type of scattering is computed using Holland's relaxation times [41]. Then, random numbers are drawn and compared to these probabilities to determine if the phonon is scattered or not during this time step. After some time, when the temperature gradient and the resulting heat flux are well established, the
TC is calculated with a Fourier formalism:

$$
\frac{\vec{J}}{S}=-\kappa \times \vec{\nabla} T,
$$

with $\vec{J}$ the heat flux obtained from energies and velocities of phonons, $S$ the cross section of the system, $\kappa$ the TC, and $\vec{\nabla} T$ the imposed temperature gradient.

In nanostructured materials like PnC membranes, phonons also experience boundary scattering at the membrane edges or at the holes surfaces. Reflections at boundaries are directly done during the displacement of the phonons. Phonons can be specularly or diffusely reflected according to Ziman's specularity parameter:

$$
p=\exp \left(\frac{-16 \pi^{2} \delta^{2}}{\lambda^{2}} \cos (\theta)\right),
$$

where $\delta$ is the surface roughness, $\lambda$ is the phonon wavelength, and $\theta$ is the incidence angle of the phonon. The probability of specular reflection $p$ is estimated every time a phonon encounters a boundary. Then a random number $R$ is drawn. If $R<p$ the phonon is specularly reflected at the boundary; otherwise it is diffusely scattered. $\delta_{\text {holes }}$ and $\delta_{\text {walls }}$ are chosen as input parameters. Thus, reflections at walls and holes can be enforced to diffuse with large roughness or specularity with $\delta=0$. In this work, reflections at holes are set to purely diffuse because the dominant phonon wavelength in silicon at room temperature is usually smaller than the hole roughness [42]. Moreover, the impact of hole specularity on the TC of PnC membranes is weak compared to the impact of membrane wall specularity, especially when the neck becomes larger than the height of the membrane [7].

More details about our Monte Carlo modeling of heat transport in nanostructures can be found in previous papers $[39,44]$.

\section{RESULTS AND DISCUSSION}

\section{A. Amorphous shells around holes}

First we investigated with molecular dynamics TC variations in silicon membranes due to cylindrical nanoholes with an amorphous-phase shell of thickness $t_{s}$ around the holes. The TC is computed in the in-plane direction of the membranes (perpendicular to the axis of the cylinder), with the EMD technique. Results are presented in Fig. 3. First we examined a PnC silicon membrane without amorphous shells around holes. Similar molecular dynamics simulations for PnC membranes without amorphous shells were performed by Lacatena et al. [6] and are added for comparison (blue triangle). The discrepancy between the two calculated TCs is small and it comes from the use of different interatomic potentials: the Stillinger-Weber potential in our study and the environment-dependent interatomic potential (EDIP) in the previous work [6]. We have to stress that the relative difference between PnC bulk (periodic boundary conditions in the $z$ direction) and membrane TCs is preserved with both interatomic potentials, when there is no amorphous shells around the holes (the TC of the PnC membrane is about $75 \%$ of the PnC bulk value). 


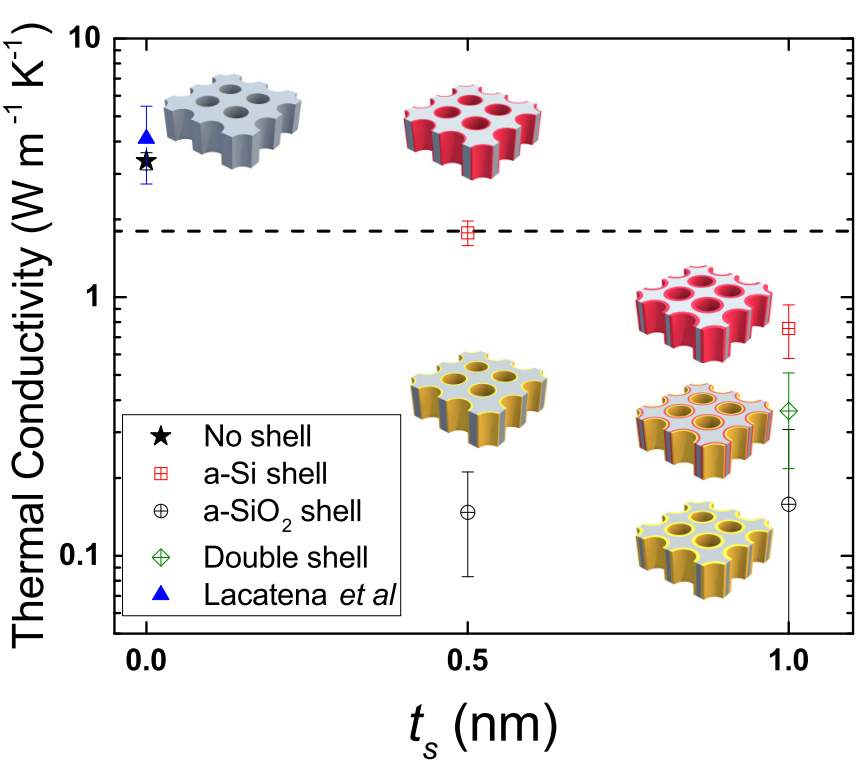

FIG. 3. In-plane TC of PnC membranes depending on the type and the thickness $t_{s}$ of the amorphous shell around the holes. The MD result with same dimensions and without amorphous shell from Lacatena et al. [6] has been added for comparison. Grey, red, and yellow correspond to the crystalline silicon, amorphous silicon, and amorphous silica, respectively (see Fig. 2). The dashed line indicates the amorphous limit of silicon $\left(1.8 \mathrm{~W} \mathrm{~m}^{-1} \mathrm{~K}^{-1}\right.$ [45]).

When there is an a-Si shell around the holes, first of all, the difference between the TC of the PnC bulk and the membrane decreases, particularly when the shell thickness is large: for PnC bulk with an a-Si shell with $t_{s}=1 \mathrm{~nm}$, the TC is found to be $0.8 \mathrm{~W} \mathrm{~m}^{-1} \mathrm{~K}^{-1}$, while it is about $0.76 \mathrm{~W} \mathrm{~m}^{-1} \mathrm{~K}^{-1}$ for a PnC membrane with the same amorphous shell thickness. Thus, the relative difference between bulk and membrane goes from $25 \%$ when there is no shell to $5 \%$ for an a-Si shell thickness of $1 \mathrm{~nm}$. This means that the characteristic lengths which control heat transport in the in-plane direction in these systems are those associated to the interfaces perpendicular to the heat flux (hole diameter and period, shell thickness), while the thickness of the membrane (geometric parameter associated to the interfaces parallel to the heat flux) does not play an important role. For $t_{s}=0.5 \mathrm{~nm}$, the TC is divided by 2 compared to the system without a shell. This reduction is more pronounced when the shell thickness is increased to $1 \mathrm{~nm}$ (reduction by a factor of 5). Subamorphous TCs are reached for $t_{s}=1 \mathrm{~nm}$. The presence of an amorphous phase in the system dramatically decreases thermal transport, as observed recently for the case of membranes without holes [22] and also in systems with spherical nanopores [23].

Systems with a-SiO 2 shells around the holes exhibit even lower TC than those with a-Si shells. The TC difference in increasing the thickness of the amorphous shell is less pronounced. A general trend is that the TC is lower when there is a native oxide compared to amorphous silicon. The TCs reached by nanostructuration and oxidation can be lower than the bulk amorphous a-SiO $\mathrm{SC}_{2} \mathrm{Tabout} 1.4 \mathrm{~W} \mathrm{~m}^{-1} \mathrm{~K}^{-1}$ [46]). This is related to supplementary scattering mechanisms introduced in the system thanks to the holes and the c-Si/a-SiO interfaces. For the systems with the double shell (both a-Si and a- $\mathrm{SiO}_{2}$ ), the TC of the PnC membrane is also ultralow. The total thickness of the double shell is $1 \mathrm{~nm}$, so it has to be compared with systems having a shell of a-Si or a-SiO $\mathrm{S}_{2}$ of $1 \mathrm{~nm}$. The $\mathrm{TC}$ is between those of a PnC membrane with an a-Si shell of $1 \mathrm{~nm}$ and of a PnC membrane with an $\mathrm{a}_{-} \mathrm{SiO}_{2}$ shell of $1 \mathrm{~nm}$, which is quite logical considering that between two amorphous materials the Kapitza resistance is negligible compared to the intrinsic thermal resistance inside both amorphous materials.

A more detailed analysis of heat carriers in amorphous layers can be conducted. The taxonomy of heat carriers in amorphous material can be achieved on the basis of their frequency $[18,47]$. For low frequencies, there are propagons, at large frequencies locons, and in between, diffusons. Heat is carried by propagons and mainly by diffusons. Yet, what is interesting to notice is that propagons can ballistically go through thin amorphous layers. This was recently demonstrated by Liang et al. [48] for $\mathrm{a}_{-} \mathrm{SiO}_{2}$ thin layers $(4.6-9.2 \mathrm{~nm})$ sandwiched between Si leads. In this work the governing criterion to check the propagative behavior of carriers is the Ioffe-Regel (IR) frequency that discriminates between propagons and diffusons. In Ref. [48] the IR frequency of a-SiO 2 is about $1.4 \mathrm{THz}$, while for a-Si it is about $2 \mathrm{THz}$ [49]. Thus, there are propagative modes on a more extended frequency range in amorphous silicon as compared to silicon dioxide. This might impact heat transport as the amorphous layers considered here are ultrathin and consequently ballistic transports within the edge of a layer can arise. Above the Ioffe-Regel frequency, carrier transmission continuously decreases and the contribution to heat transport is equivalent for both a-Si and $\mathrm{a}-\mathrm{SiO}_{2}$.

To complete these results, a discussion about the impact of the different amorphous shells on the phonon DOS of the PnC is given in Appendix A. In general the creation of the holes does not impact the shape of the DOS; it only decreases the relative populations of the transversal acoustic (TA) and longitudinal optic (LO) modes. Adding amorphous shells around the pores causes the peaks of all modes to be shifted toward lower frequencies and flattened, while increasing the amorphous shell thickness yields the same DOS of the system as that of bulk amorphous phase.

The softening of the modes can be explained partially by the enhancement of anharmonicity due to the existence of the amorphous phase and the amorphous/crystalline interfaces. Other reasons for this softening are the reduced periodicity which might eliminate the Van Hove singularities [50], the increase of the surface modes, and the drastic decrease of the phonon group velocities [51]. He et al. [51] studied nanostructures similar to our PnC membranes with and without an a-Si shell by means of molecular and lattice dynamics considering much smaller porosities (7-9\%). They pointed out that the important decrease of the $\mathrm{TC}$ is related to the one-order-of-magnitude decrease of the phonon group velocity in the in-plane direction perpendicular to the holes. Finally, amorphization could also reduce the phonon lifetimes.

\section{B. Hole diameter and period}

\section{Variation of hole diameter}

From now on, the study is focused only on PnC membranes with a-Si shells. Although the systems with oxidation are more realistic, for reasons of computational resources due 

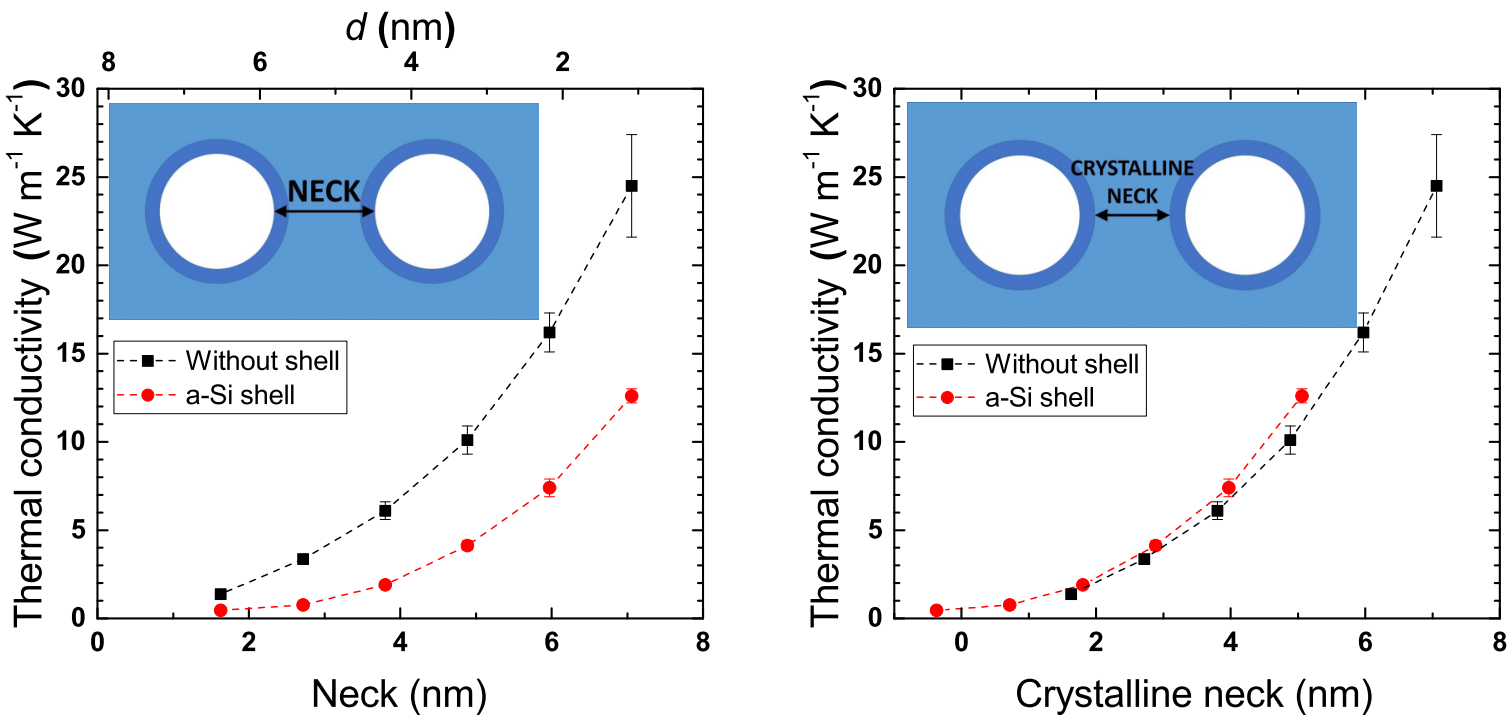

FIG. 4. TC dependence on the nanohole size. Left: TC as a function of the neck and the hole diameter. Right: TC as a function of the "crystalline neck." The neck is the distance between the edges of two neighboring holes (for systems with a-Si shells, the shells are contained in the neck). The crystalline neck is the width of the path of pure c-Si between two neighboring holes or shells.

to the use of the Tersoff potential and the stability of the autocorrelation function, we were obliged to continue the study with a-Si instead of $\mathrm{a}_{-} \mathrm{SiO}_{2}$. We believe that this approximation is not so severe to draw conclusions from as a-Si and a- $\mathrm{SiO}_{2}$ are amorphous phases with $\mathrm{TC}$ of 1.4 and $1.8 \mathrm{~W} \mathrm{~m}^{-1} \mathrm{~K}^{-1}$, respectively, and two orders of magnitude lower than the TC of bulk c-Si $\left(\sim 150 \mathrm{~W} \mathrm{~m}^{-1} \mathrm{~K}^{-1}\right)$. Moreover, it was shown in Sec. III A that a-Si and a-SiO ${ }_{2}$ have a similar impact on the TC of PnCs. According to results from Fig. 3, one should expect that systems with a-SiO ${ }_{2}$ would have slightly smaller TC than systems with a-Si. The studied parameter in this section is the diameter of the nanohole, and thus the porosity, while the dimensions of the systems are the same as previously $\left(h=15 a_{0}\right.$ and $a=15 a_{0}=8.145 \mathrm{~nm}$ ).

In Fig. 4 (left), the in-plane TC as a function of the neck size (the distance between the edges of two neighboring holes) and the hole diameter is depicted. Even for very small diameter $\left(d=2 a_{0}=1.086 \mathrm{~nm}\right)$, the existence of an amorphous shell $\left(\mathrm{a}-\mathrm{Si}, t_{s}=1 \mathrm{~nm}\right)$ reduces the TC by a factor of 2 compared to $\mathrm{PnC}$ membranes without amorphous shells. Moreover, we can see from Table II that the relative reduction of the TC

TABLE II. In-plane TC of PnC membranes with same period ( $\left.a=15 a_{0}=8.145 \mathrm{~nm}\right)$ but different hole sizes, with and without an a-Si shell around the hole. The relative difference between TCs of systems with and without shells is also indicated.

\begin{tabular}{|c|c|c|c|c|c|}
\hline \multirow{2}{*}{$\begin{array}{l}d \\
\left(a_{0}\right)\end{array}$} & \multirow{2}{*}{$\begin{array}{l}\text { Neck } \\
\left(a_{0}\right)\end{array}$} & \multirow{2}{*}{$\begin{array}{c}\text { Porosity } \\
\text { (\%) }\end{array}$} & \multicolumn{2}{|c|}{$\mathrm{TC}\left(\mathrm{W} \mathrm{m} \mathrm{m}^{-1} \mathrm{~K}^{-1}\right)$} & \multirow{2}{*}{$\begin{array}{c}\text { Relative } \\
\text { difference }(\%)\end{array}$} \\
\hline & & & Without shell & a-Si shell & \\
\hline 2 & 13 & 1.4 & $24.5 \pm 2.9$ & $12.6 \pm 0.4$ & $49 \pm 8$ \\
\hline 4 & 11 & 5.6 & $16.2 \pm 1.1$ & $7.4 \pm 0.5$ & $54 \pm 6$ \\
\hline 6 & 9 & 12.6 & $10.1 \pm 0.8$ & $4.1 \pm 0.3$ & $59 \pm 6$ \\
\hline 8 & 7 & 22.3 & $6.1 \pm 0.5$ & $1.9 \pm 0.1$ & $69 \pm 5$ \\
\hline 10 & 5 & 34.9 & $3.4 \pm 0.3$ & $0.8 \pm 0.2$ & $77 \pm 7$ \\
\hline 12 & 3 & 50.3 & $1.4 \pm 0.1$ & $0.45 \pm 0.03$ & $67 \pm 4$ \\
\hline
\end{tabular}

due to the amorphous shell is getting larger with increasing diameter of the holes. This can be easily understood in terms of the reduction of the neck size. When the diameter increases while the period does not change, the neck decreases and the fraction of the neck which is "cropped" by the amorphous shell of thickness $1 \mathrm{~nm}$ becomes more important. For instance, for the smallest diameter, $d=2 a_{0}=1.086 \mathrm{~nm}$, the neck is $n=a-d=15-2=13 a_{0} \simeq 7 \mathrm{~nm}$. So for the system without the shell, there is a crystalline path with width of $7 \mathrm{~nm}$ between holes, while for systems with the shell, $2 \mathrm{~nm}$ of the neck is replaced by amorphous silicon, so the width of the crystalline phase between two holes ("crystalline neck") now is only $5 \mathrm{~nm}$. Interestingly, the crystalline neck is the parameter which controls heat transport in such systems; this can be seen in Fig. 4 (right), where the TC is plotted for both systems (with and without shell) as a function of the crystalline neck. The two curves are almost superposed. This confirms a trend already observed in systems with spherical nanopores [23]: amorphous silicon shells around pores inside a crystalline silicon matrix have almost the same impact on thermal transport as voids (pores or holes).

\section{Variation of hole period}

In this part, we examine the impact of the period on the TC of PnC membranes. To do so, the diameter of the hole is set constant to $10 a_{0}=5.43 \mathrm{~nm}$, and it is surrounded or not by an a-Si shell with $t_{s}=1 \mathrm{~nm}$; the height of the membrane is $15 a_{0}=$ $8.145 \mathrm{~nm}$, while the period of the structure (the distance center to center between two neighboring holes) varies from $15 a_{0}$ to $25 a_{0}(8.145-13.575 \mathrm{~nm})$. The TC of a membrane of the same height without holes has also been computed to achieve a comparison. The TC of PnC membranes should be the same as the plain membrane when the period becomes large enough. MD results are shown in Table III and in the last section in Fig. 8, in which these results are compared to MC simulations.

For a given period, systems with a-Si shells always have lower TC than analogous systems without shells. The relative 
TABLE III. In-plane TC of PnC membranes with same hole diameter but different periods, with and without a-Si shell around the holes.

\begin{tabular}{lccr}
\hline \hline Period & Porosity & \multicolumn{2}{c}{$\mathrm{TC}\left(\mathrm{W} \mathrm{m}^{-1} \mathrm{~K}^{-1}\right)$} \\
\cline { 3 - 4 }$\left(a_{0}\right)$ & $(\%)$ & Without shell & $\mathrm{a}-\mathrm{Si}$ shell \\
\hline Membrane & 0 & $53 \pm 5$ & \\
25 & 12.5 & $15.2 \pm 1.9$ & $9.0 \pm 0.9$ \\
20 & 20 & $8.6 \pm 0.5$ & $4.4 \pm 0.3$ \\
15 & 35 & $3.4 \pm 0.3$ & $0.8 \pm 0.2$ \\
\hline \hline
\end{tabular}

reduction due to the existence of an amorphous shell is more important when the period is small as expected, with the volume fraction of the amorphous compound getting larger. For the largest period, the TC of the structure with the shell is lowered by $40 \%$ compared to the no-shell system, although the amorphous volume fraction in the system is only $10 \%$. For the smallest period, the TC with shell is merely $25 \%$ of the TC without shell.

\section{Amorphization of external membrane surfaces with and without holes}

The recent work of Neogi et al. [22] showed an important decrease of the TC, of the order of 2, due to the native oxide on the membranes. In this section, the impact of the amorphous silicon on the edge of plain membranes and then on $\mathrm{PnC}$ membranes with and without amorphous shells around the holes is studied. In this case, these amorphous layers on the two sides of the membranes are parallel to the heat flux and we see that their impact on thermal transport is less pronounced compared to the amorphous shells around holes.

\section{Plain membrane with amorphous edges}

First, we consider a plain membrane with amorphous layers on external surfaces (see left schematic membrane in Fig. 6). There are two ways to consider the amorphous phase at the edge of the crystalline membrane in our EMD simulations:

(1) The amorphous silicon layer can be added "outside" the membrane, keeping a crystalline thickness of $15 a_{0}$ $(8.145 \mathrm{~nm})$ and increasing the total height of the membrane.

(2) The amorphous silicon layer can be added "inside" the membrane, replacing a part of the crystalline phase. In the latter case, the total height of the membrane is preserved while the crystalline thickness is reduced. This corresponds to thin membranes of c-Si which are experimentally elaborated, where the measured thickness includes the amorphous layer.

These two cases have been investigated on the $15 a_{0}$ $(8.145 \mathrm{~nm})$ height membrane with molecular dynamics and the equilibrium methodology. The thickness of the amorphous layer $t_{e}$ was $1 a_{0}$ or $2 a_{0}(0.543$ or $1.086 \mathrm{~nm}$, respectively). The computed TC of each system is presented in Table IV and in Fig. 5.

Surprisingly, adding a-Si outside, even if the membrane is getting thicker, the TC is reduced by $40 \%$ compared to the nonamorphized membrane (black curve in Fig. 5). Recent works show that the amorphous phase modifies the phonon DOS of crystalline silicon close to the amorphous/crystalline
TABLE IV. In-plane TC of plain membranes with amorphous edges. The first row corresponds to a pristine crystalline membrane of height $h=8.145 \mathrm{~nm}$.

\begin{tabular}{lcc}
\hline \hline a-Si localization & $t_{e}(\mathrm{~nm})$ & $\mathrm{TC}\left(\mathrm{W} \mathrm{m}^{-1} \mathrm{~K}^{-1}\right)$ \\
\hline None & 0 & $53 \pm 5$ \\
Outside & 0.543 & $32.0 \pm 3.7$ \\
Outside & 1.086 & $27.1 \pm 2.1$ \\
Inside & 0.543 & $26.3 \pm 2.2$ \\
Inside & 1.086 & $19.0 \pm 1.5$ \\
\hline \hline
\end{tabular}

interface up to $0.8 \mathrm{~nm}$ from the interface [20]. However, this alone cannot explain the amplitude of the TC reduction, because there is still a thickness of $6.5 \mathrm{~nm}$ of c-Si with bulk c-Si DOS (with our EMD methodology, the thermal conductivity of a pure crystalline silicon membrane with height of $6.5 \mathrm{~nm}$ is found to be about $49 \mathrm{~W} \mathrm{~m}^{-1} \mathrm{~K}^{-1}$ ). In fact, adding a-Si introduces an artificial interface roughness in our MD simulations which further hinders thermal transport inside the crystalline part of the membrane, as discussed in the last section. Considering amorphization of the membrane edges, the calculated TC is close to what has been experimentally measured for ultrathin membranes (below $20 \mathrm{~W} \mathrm{~m}^{-1} \mathrm{~K}^{-1}$ [52]). When the thickness of the amorphous layer is increased, the TC is slightly further reduced. This "extra" reduction is not due to an increase of roughness but can rather be explained by the larger fraction of amorphous material in the structure. We can also make the hypothesis that it is linked to the presence of a Kapitza-like resistance parallel to the flux which appears between the crystalline and amorphous phases.

Adding a-Si edges inside the membrane, the reduction of the TC is even more pronounced (red curve in Fig. 5). Besides creating an interface roughness, a part of the crystalline path for phonons is replaced by a-Si. The TC is lower when the

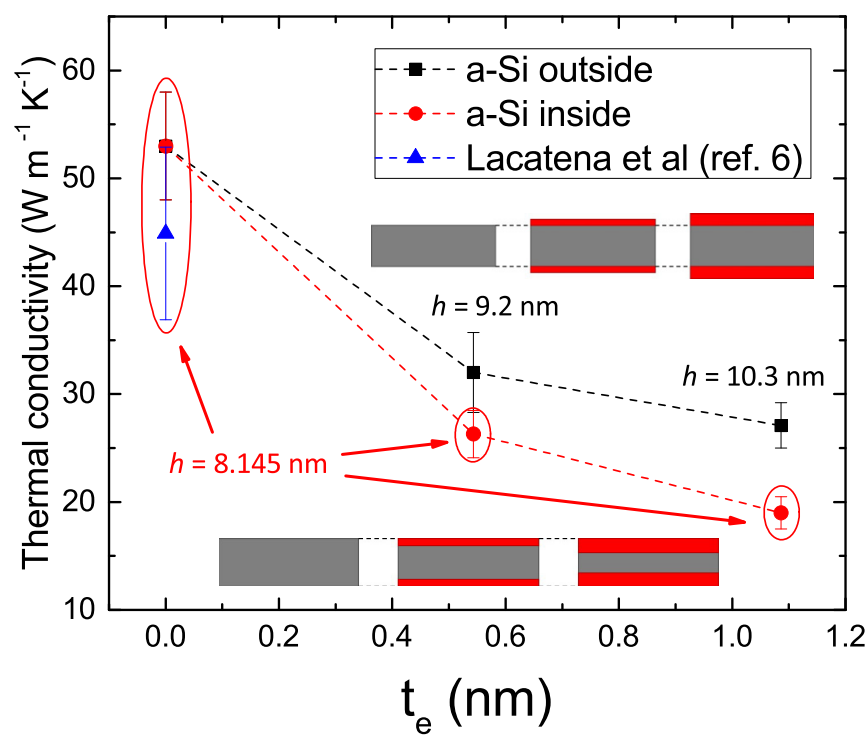

FIG. 5. TC of silicon plain membranes with or without amorphous edges as a function of the amorphous layer thickness $t_{e} . h$ is the total height of the membrane, including both amorphous and crystalline phases. 

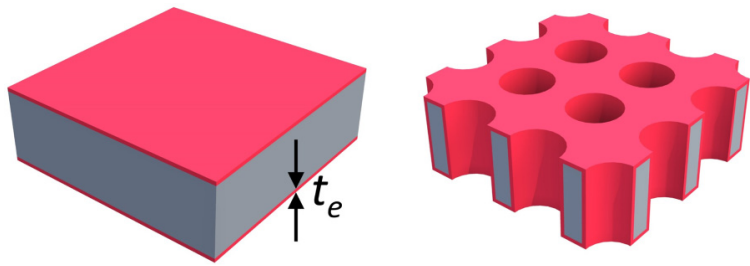

FIG. 6. Silicon membranes with amorphous edges (red layers). Left: Plain membrane. Right: PnC membrane.

amorphous thickness increases. It should tend to the TC of a totally amorphous membrane when the amorphous thickness is increased further. With $t_{e}=2 a_{0}(1.086 \mathrm{~nm})$, the TC becomes very close to experimental measurements and is only $36 \%$ of the TC of the nonamorphized membrane. The free surfaces of crystalline membranes often become amorphous with the formation of silicon dioxide when they are exposed to air, as can be seen in Fig. 1 and in the existing literature [22]. Adding a-Si edges inside the membrane in simulations corresponds to the experimental case, where the measured thickness is the total one, including the a-Si layer. This might explain why the TCs experimentally measured on thin membranes are so low.

\section{PnC membrane with amorphous edges and hole shells}

Finally, membranes with a-Si edges and cylindrical nanoholes with a-Si shells around them have been modeled (see right schematic membrane in Fig. 6). The height of the membrane is still $15 a_{0}(8.145 \mathrm{~nm})$ and the amorphous edges are added "inside" the system (the total height of the membrane is preserved). The diameter of the holes is $d=10 a_{0}(5.43 \mathrm{~nm})$, so TCs of theses structures can be compared to those of PnC membranes without amorphous external layers (systems from Sec. III A). Results are gathered in Table V and in Fig. 7.

When amorphous edges are added in addition to the amorphous shells, the TC is further decreased by $20 \%$. The reduction is much less pronounced than for plain membranes with amorphous edges inside the system. This lowering of thermal transport is primarily related to the decrease of the crystalline volume fraction compared to the total one, taking into account the holes. Furthermore, the diffuse scattering of phonons on the vertical walls of the holes is enhanced while increasing the surface area between the crystalline and the amorphous phases. Comparing the first and last lines of Table V, the TC of PnC membranes can be divided by 6 if a layer of about $1 \mathrm{~nm}$ of amorphous silicon appears at both the surface of the holes and the membrane edges.

TABLE V. In-plane TC of PnC membranes with amorphous edges and an amorphous shell around the hole.

\begin{tabular}{lcc}
\hline \hline$t_{s}(\mathrm{~nm})$ & $t_{e}(\mathrm{~nm})$ & $\mathrm{TC}\left(\mathrm{W} \mathrm{m}^{-1} \mathrm{~K}^{-1}\right)$ \\
\hline 0 & 0 & $3.36 \pm 0.26$ \\
0.543 & 0 & $1.78 \pm 0.20$ \\
1.086 & 0 & $0.76 \pm 0.18$ \\
0.543 & 0.543 & $1.52 \pm 0.11$ \\
1.086 & 1.086 & $0.57 \pm 0.21$ \\
\hline \hline
\end{tabular}

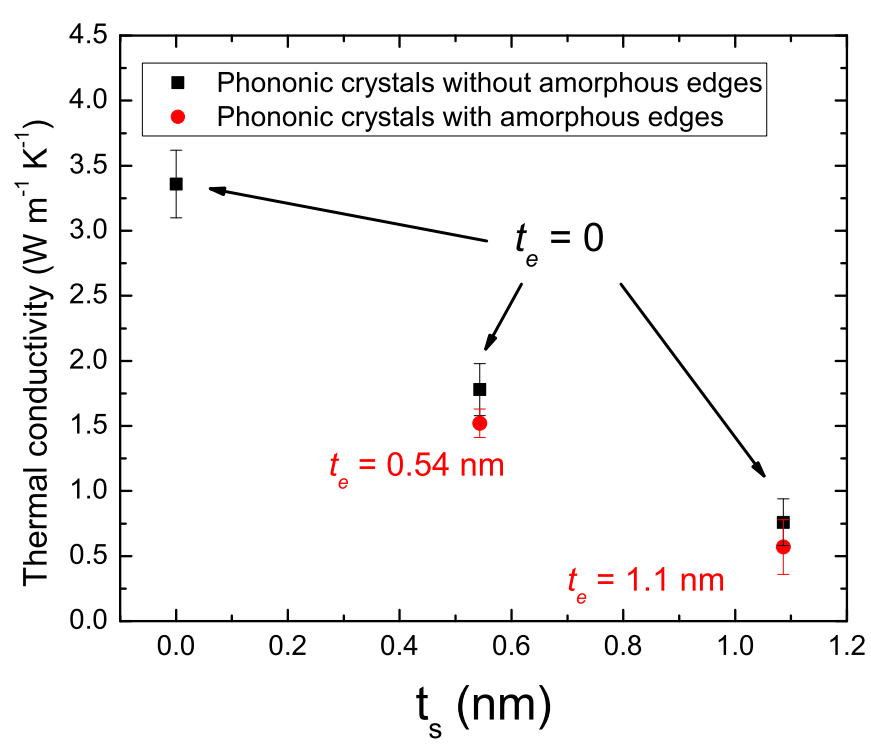

FIG. 7. TC of silicon PnC membranes as a function of the thickness of the amorphous shell around the holes, $t_{s} . t_{e}$ is the thickness of the amorphous layer at the edges of the membranes (added inside, so the total membrane height is preserved: $h=8.145 \mathrm{~nm}$ ).

\section{Specularity investigation with Monte Carlo modeling}

In this section, the MC method is used to retrieve the TCs of plain and PnC membranes obtained from MD simulations. The TC of the plain membrane given by $\mathrm{MD}$ is about $53 \mathrm{~W} \mathrm{~m}^{-1} \mathrm{~K}^{-1}$ (see Sec. III B 2), which seems surprisingly high as the thickness of the membrane is only $8.145 \mathrm{~nm}\left(15 a_{0}\right)$. Experimental data from the literature [52] give $\kappa \simeq 9 \mathrm{~W} \mathrm{~m}^{-1} \mathrm{~K}^{-1}$ for a membrane of thickness $h \simeq 9 \mathrm{~nm}$. This value follows the decreasing trend of the TC when the height of the membrane is reduced. Other studies conducted with MD also reveal a surprisingly large TC for silicon membranes. Lacatena et al. computed the TC of the same membrane with a different interatomic potential (EDIP) and found $\kappa=44.9 \mathrm{~W} \mathrm{~m}^{-1} \mathrm{~K}^{-1}$ [6]. Neogi et al. used EMD and the Tersoff potential to find the thermal conductivity of silicon plain membranes. With smooth surfaces and no oxide in the system, the thermal conductivity reached $\sim 115 \mathrm{~W} \mathrm{~m}^{-1} \mathrm{~K}^{-1}$ for a membrane height of $\sim 10 \mathrm{~nm}$.

In order to assess the importance of boundary conditions and especially the transition between specular and diffuse scattering at the membrane walls, we have introduced a specularity parameter $p$ in $\mathrm{MC}$ simulations to control phonon reflections at boundaries (see Sec. II C for details). With fully diffuse membrane walls, the TC of PnCs and plain membranes given by $\mathrm{MC}$ is lower than that obtained from $\mathrm{MD}$, as can be seen in Fig. 8. With MC, the TC of the plain membrane is about $13 \mathrm{~W} \mathrm{~m}^{-1} \mathrm{~K}^{-1}$, which is close to the experimental value. The further reduction of thermal transport experimentally observed could result from the amorphization of the edges, which is not modeled in MC simulations.

In order to retrieve the MD thermal conductivity of the plain membrane, we varied the walls surface roughness $\delta_{\text {walls }}$ in MC simulations. The MC thermal conductivity reaches $55 \mathrm{~W} \mathrm{~m}^{-1}$ $\mathrm{K}^{-1}$ for $\delta_{\text {walls }}=0.34 \pm 0.04 \AA$ (see Appendix B for details). This value is unrealistic because it is less than an interatomic 


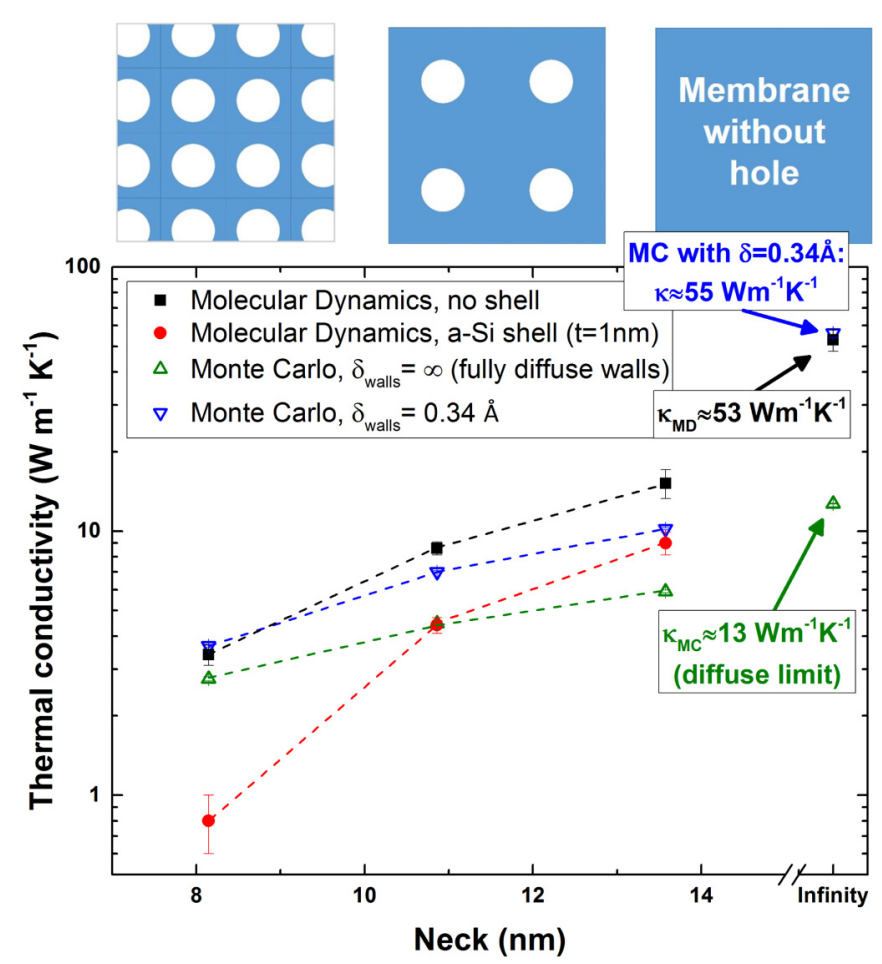

FIG. 8. TC of PnC membranes as a function of the neck. MD results come from Table III. MC simulations were first performed with diffuse reflections on membrane walls (green upward triangles), then with a specularity parameter in order to align with MD results (blue downward triangles).

distance. But during MD simulations, surfaces likely have a nonzero but unknown surface roughness [53]. In fact, there are two ways to consider the atomic surface roughness in MD:

(1) It can be defined as the distance between the two first atomic layers near the surface [Fig. 9(a)]. With crystalline silicon, this definition gives $\delta_{\text {walls }} \simeq 1.4 \AA$.

(2) It can also be defined as the rms roughness of the first layer of atoms [Fig. 9(b)] averaged over all the simulation duration. This has been computed for one MD simulation and we obtained $\delta_{\text {walls }}=0.38 \pm 0.04 \AA$, which is compatible with the fitted value of MC simulations ( $\delta_{\text {walls }}=0.34 \pm 0.04 \AA$ ).

So we can make the hypothesis that molecular dynamics overestimates the TC of thin silicon membranes because

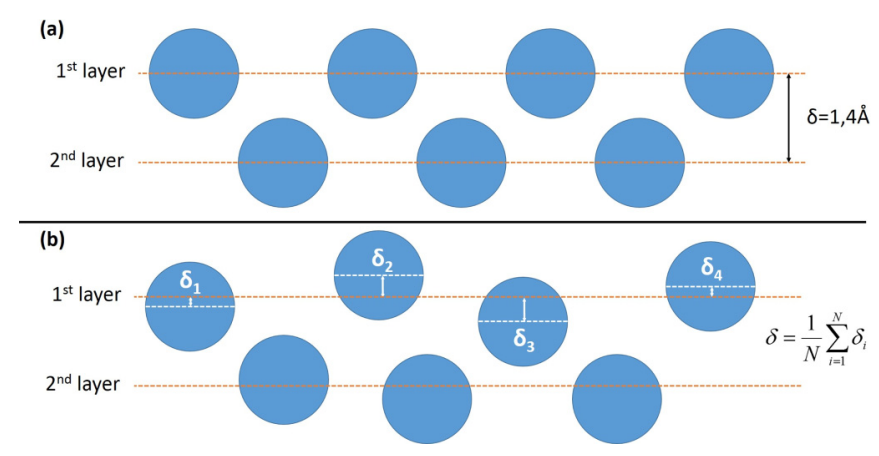

FIG. 9. Two possible definitions of membrane surface roughness during MD simulations. We recall that the growth of silicon membranes is in the [001] direction. it models a too-small artificial surface roughness of the membrane walls $\delta_{\text {walls }} \simeq 0.38 \AA$, which is based on the rms roughness of the first atomic layer near the surface. With such a low roughness, most of the phonon reflections on membrane walls are specular and the boundary scattering does not reduce the TC a lot, as the walls are parallel to the direction of measurement (in-plane direction) [44]. Adding a-Si outside the membrane could create an artificial roughness greater than $0.38 \AA$, which reinforces diffuse reflections. This can explain the strong reduction of thermal transport observed with $\mathrm{MD}$ in previous sections when adding a-Si outside the plain membrane. Similarly, oxidation of the edges of elaborated membranes which have been exposed to air surely leads to more diffusive scattering of phonons and lower thermal conductivity. Moreover, we have seen in Sec. III A that the occurrence of $\mathrm{a}_{-} \mathrm{SiO}_{2}$ in $\mathrm{PnCs}$ reduces thermal transport more than the occurrence of a-Si. Thus, the discrepancy between experimental measurements and numerical results could be explained by the oxidation, without considering coherent effects or unknown physical phenomena related to the patterning of the PnC.

\section{CONCLUSIONS}

The impact of amorphous phases around the holes and at the upper and lower free surfaces on thermal transport in silicon PnC membranes has been studied. The reduction of the TC due to silicon dioxide is greater than that due to amorphous silicon. Increasing the thickness of the amorphous shells of holes or edges of membranes (exposure of the membranes to nonprotective conditions) leads to an important decrease of the TC, even to ultralow subamorphous thermal conductivity keeping a large fraction of crystalline material. We found also that, when an amorphous phase is added, the key parameter to control heat transfer is the crystalline neck distance and not the distance between the two voids. Decreasing the neck distance between holes varying their diameter or their period results in a sharp decrease of the TC (40-75\% in the present work). This effect is more pronounced for PnCs with small dimensions, particularly when the neck is small. We have shown that external amorphous surfaces of membranes reduce further the $\mathrm{TC}$ for both plain membranes and $\mathrm{PnC}$ ones. Interestingly, when the crystalline thickness of the membrane is kept constant and thicker external amorphous layers are added, the TC decreases sharply. That means that phonons become more confined in the same crystalline volume when amorphous material is added around them. Subamorphous TC appears in several studied systems, making phonon engineering possible upon controlling the native oxides.

Comparing the results of EMD and MC, we found that the specularity of the free surfaces parallel to the heat flux, which is linked to the surfaces roughness, is an important parameter to take into account for the modeling of membranes. For PnCs, the roughness of free surfaces has a weak impact on the thermal conductivity because the reduction of thermal transport is mainly due to the holes, which are perpendicular to the direction of interest. Nevertheless, for in-plane thermal conductivity of plain membranes, all the surfaces are parallel to the heat flux and one should add an artificial roughness in MD simulations, amorphizing or oxidizing the membrane edges, 


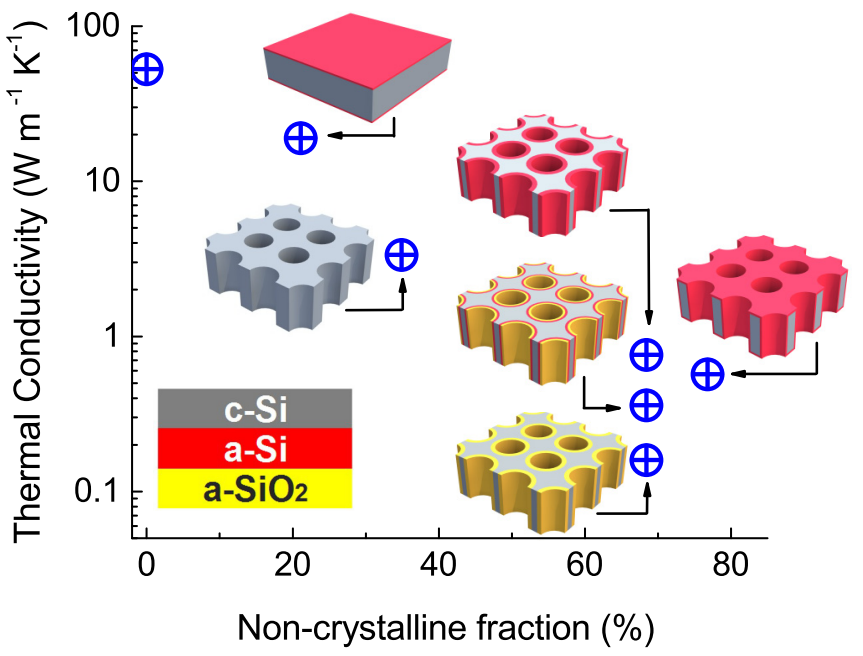

FIG. 10. Thermal conductivity of plain membrane and PnCs with and without amorphous shells and edges as a function of the noncrystalline fraction. The height of each membrane is $h=8.145 \mathrm{~nm}$. The diameter of the hole for each PnC is $d=5.43 \mathrm{~nm}$. The thickness of amorphous shells for PnCs with shells is $t_{s} \sim 1 \mathrm{~nm}$ and the thickness of amorphous edges for the membrane and the PnCs which have amorphous edges is $t_{e} \sim 1 \mathrm{~nm}$.

for instance, to obtain better agreement with experimental measurements.

Finally, with this study, we can define a hierarchy of the impact of the orientation and the nature of interfaces on the reduction of thermal transport as compared to the plain membrane. At the scale studied in the present work, the most important reduction is due to the surfaces of the holes, which are perpendicular to the in-plane direction. Just creating cylindrical holes of diameter of $10 a_{0}$ with a period of $15 a_{0}$ in the membrane, the TC is lowered by $\sim 92 \%$ compared to the plain membrane. Then, the second most important reduction ( $\sim 70 \%$ ) comes from the existence of amorphous shells around the holes. The least influence comes from the amorphization of the edges of the membranes (with or without holes) and is estimated to be of the order of $\sim 50 \%$. In contrast to the two previous cases, the amorphous layers at the edges of the membranes are parallel to the in-plane direction, which explains their smaller impact on the heat transport. In Fig. 10 we gather results for all cases studied here as a function of the noncrystalline fraction. Ultralow thermal conductivity can be observed for crystalline phononic crystals with porosity of $30 \%$, while subamorphous thermal conductivity can be obtained with the amorphization of holes and/or edges, keeping a crystalline fraction of $30 \%$.

\section{ACKNOWLEDGMENTS}

The authors would like to acknowledge to R. Jucquin (Lorraine University) and Dr. A. France-Lanord (CEA Material Design) for many helpful discussions throughout this study. Calculations were partly performed on the large computer facilities GENCI-IDRIS (Grant No. 2018-A0030907186) and on the EXPLOR Mesocenter (University of Lorraine). The research leading to these results has received funding from the
European Research Council under the European Community's Seventh Framework Programme (FP7/2007-2013) ERC Grant No. 338179. The authors acknowledge support from the STMicroelectronics-IEMN common laboratory and the French RENATECH network.

\section{APPENDIX A}

Using molecular dynamics, the phonon DOS of the system can also be extracted from the trajectory of the atoms. Indeed, the velocity autocorrelation functions can be analyzed by Fourier transform to find the density of frequencies of phonons [54]. In this work, the specden plugin of the VMD Signal Processing Plugin Package [55] was used to calculate the phonon DOS of the PnC membranes. The trajectories of the atoms were saved from molecular dynamics simulations every 12.5 fs for a total duration of $5 \mathrm{ps}$.

Figure 11 shows the impact of the nanostructuration on the DOS of the system. In Fig. 11(a), the DOS of the crystalline silicon membranes with and without holes are given with the DOS of bulk crystalline silicon for comparison. Between the bulk and the plain (unpatterned) membrane there are not significant changes except for a small lowering of the transversal optic (TO) peak. This decrease is much more
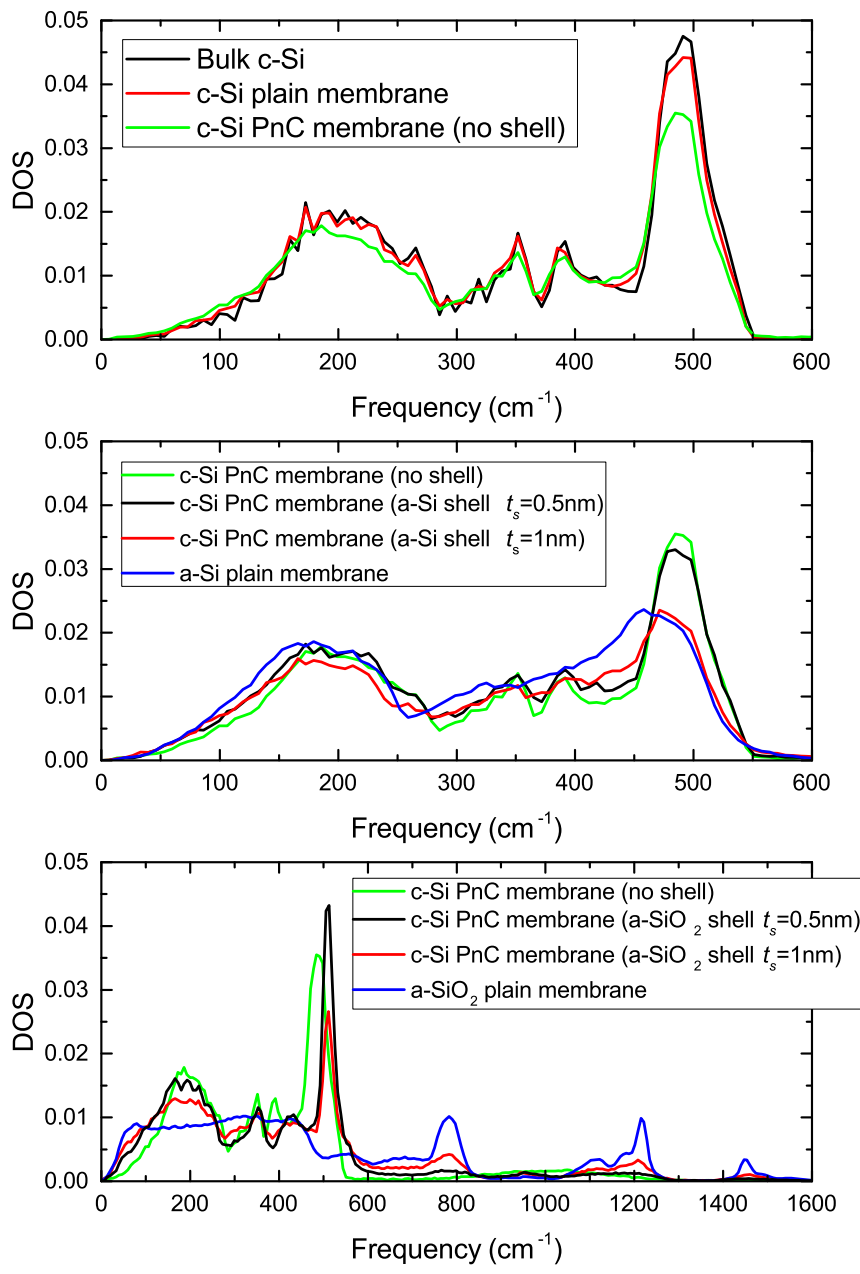

FIG. 11. Comparison of the total phonon density of states of several systems. 


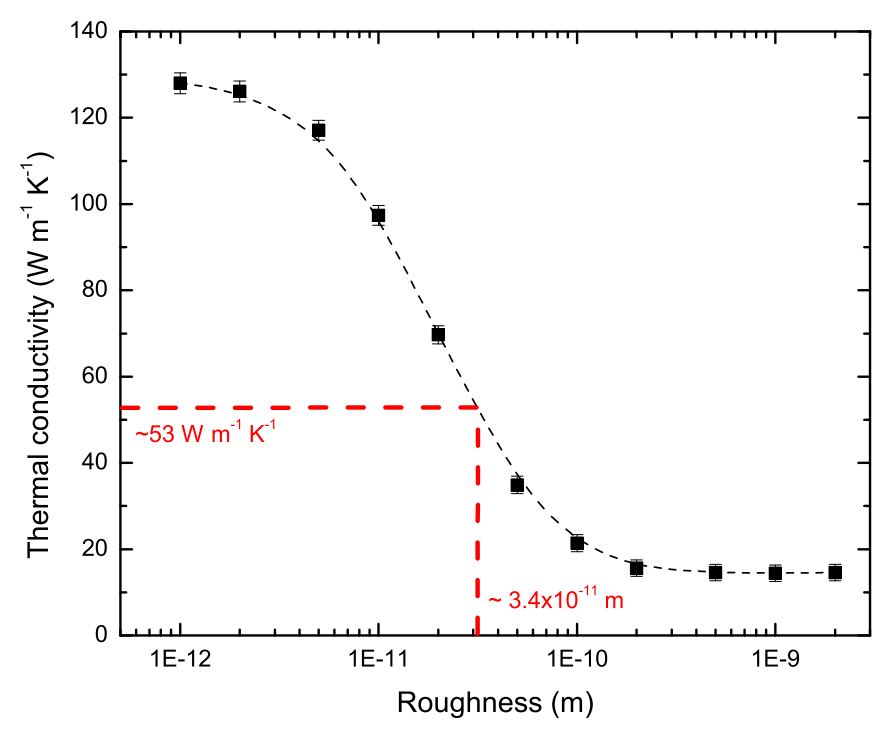

FIG. 12. Thermal conductivity computed with Monte Carlo for a plain membrane $(h=8.145 \mathrm{~nm})$ as a function of the membrane roughness.

pronounced when holes are present in the system, but it is not the cause of the great reduction of the TC, as optical phonons play a minor role in heat transport in silicon [56]. In Fig. 11(b), the effect of the thickness of the amorphous silicon shell around the holes is depicted and for the sake of comparison the DOS of crystalline and amorphous silicon membranes with same thickness are given. In increasing the thickness of the amorphous shell the TO and TA modes are redshifted (toward lower frequencies) and there is a smoothing of the two peaks in the middle, which correspond to longitudinal acoustic (LA) and LO polarizations. We can also observe in Fig. 11(b) that the DOS of the membranes with amorphous shells of $1 \mathrm{~nm}$ starts to be similar to that of the amorphous silicon membranes. In Fig. 11(c), the DOS of systems with silicon dioxide around the holes is plotted. All modes of crystalline silicon are less populated in adding a- $\mathrm{SiO}_{2}$ and a redshift is observed for the TA mode. Because of the presence of oxygen, three high-frequency peaks appear with the a-SiO 2 shell.

\section{APPENDIX B}

The thermal conductivity obtained with the MC method for a plain membrane with $h=8.145 \mathrm{~nm}$ is plotted as a function of the roughness of the membrane edges in Fig. 12. When the membrane edges are fully diffusive (when the roughness is large), the thermal conductivity is lowered to $13 \mathrm{~W} \mathrm{~m}^{-1} \mathrm{~K}^{-1}$. For very small roughness $\left(<10^{-12} \mathrm{~m}\right)$, the membrane edges become totally specular and the thermal conductivity tends to that of bulk silicon $\left(\sim 150 \mathrm{~W} \mathrm{~m}^{-1} \mathrm{~K}^{-1}\right)$ because there is no more resistive mechanism due to the nanostructuration. The thermal conductivity found with molecular dynamics $\left(\sim 53 \mathrm{~W} \mathrm{~m}^{-1} \mathrm{~K}^{-1}\right)$ corresponds to a membrane roughness of $\sim 0.34 \AA$.
[1] M. R. Wagner, B. Graczykowski, J. S. Reparaz, A. El Sachat, M. Sledzinska, F. Alzina, and C. M. Sotomayor Torres, Nano Lett. 16, 5661 (2016).

[2] P. E. Hopkins, L. M. Phinney, P. T. Rakich, R. H. Olsson, and I. El-Kady, Appl. Phys. A 103, 575 (2011).

[3] P. E. Hopkins, C. M. Reinke, M. F. Su, R. H. Olsson, E. A. Shaner, Z. C. Leseman, J. R. Serrano, L. M. Phinney, and I. El-Kady, Nano Lett. 11, 107 (2011).

[4] N. Zen, T. A. Puurtinen, T. J. Isotalo, S. Chaudhuri, and I. J. Maasilta, Nat. Commun. 5, 3435 (2014).

[5] M. Nomura, J. Nakagawa, Y. Kage, J. Maire, D. Moser, and O. Paul, Appl. Phys. Lett. 106, 143102 (2015).

[6] V. Lacatena, M. Haras, J.-F. Robillard, S. Monfray, T. Skotnicki, and E. Dubois, Appl. Phys. Lett. 106, 114104 (2015).

[7] M. Verdier, R. Anufriev, A. Ramiere, K. Termentzidis, and D. Lacroix, Phys. Rev. B 95, 205438 (2017).

[8] J. Tang, H.-T. Wang, D. H. Lee, M. Fardy, Z. Huo, T. P. Russell, and P. Yang, Nano Lett. 10, 4279 (2010).

[9] S. Kwon, M. C. Wingert, J. Zheng, J. Xiang, and R. Chen, Nanoscale 8, 13155 (2016).

[10] S. Alaie, D. F. Goettler, M. Su, Z. C. Leseman, C. M. Reinke, and I. El-Kady, Nat. Commun. 6, 7228 (2015).

[11] T. A. Puurtinen and I. J. Maasilta, Crystals 6, 72 (2016).

[12] J. Nakagawa, Y. Kage, T. Hori, J. Shiomi, and M. Nomura, Appl. Phys. Lett. 107, 023104 (2015).

[13] E. Dechaumphai and R. Chen, J. Appl. Phys. 111, 073508 (2012).
[14] J. Lee, J. Lim, and P. Yang, Nano Lett. 15, 3273 (2015).

[15] M. Nomura, J. Nakagawa, K. Sawano, J. Maire, and S. Volz, Appl. Phys. Lett. 109, 173104 (2016).

[16] J. Maire, R. Anufriev, R. Yanagisawa, A. Ramiere, S. Volz, and M. Nomura, Sci. Adv. 3, e1700027 (2017).

[17] R. Yanagisawa, J. Maire, A. Ramiere, R. Anufriev, and M. Nomura, Appl. Phys. Lett. 110, 133108 (2017).

[18] K. Termentzidis, Nanostructured Semiconductors: Amorphization and Thermal Properties (Pan Stanford Publishing, Singapore, 2017).

[19] T. Damart, V. M. Giordano, and A. Tanguy, Phys. Rev. B 92, 094201 (2015).

[20] A. France-Lanord, S. Merabia, T. Albaret, D. Lacroix, and K. Termentzidis, J. Phys.: Condens. Matter 26, 355801 (2014).

[21] A. Giri, J. L. Braun, and P. E. Hopkins, J. Appl. Phys. 119, 235305 (2016).

[22] S. Neogi, J. S. Reparaz, L. F. C. Pereira, B. Graczykowski, M. R. Wagner, M. Sledzinska, A. Shchepetov, M. Prunnila, J. Ahopelto, C. M. Sotomayor-Torres, and D. Donadio, ACS Nano 9, 3820 (2015).

[23] M. Verdier, K. Termentzidis, and D. Lacroix, J. Appl. Phys. 119, 175104 (2016).

[24] V. Lacatena, M. Haras, J.-F. Robillard, S. Monfray, T. Skotnicki, and E. Dubois, Microelectron. Eng. 121, 131 (2014).

[25] J. Trasobares, F. Vaurette, M. Francois, H. Romijn, J.-L. Codron, D. Vuillaume, D. Théron, and N. C. Beilstein, J. Nanotechnol. 5, 1918 (2014). 
[26] The starting point of the process is a silicon-on-insulator with a 70- or 55-nm-thick silicon layer and a $145-\mathrm{nm}$ buried oxide layer (BOX). After development the pattern is transferred to the silicon layer by means of reactive ion etching (RIE) of $\mathrm{Si}$ with $\mathrm{Cl}_{2}$ gas.

[27] A. France-Lanord, E. Blandre, T. Albaret, S. Merabia, D. Lacroix, and K. Termentzidis, J. Phys.: Condens. Matter 26, 055011 (2014).

[28] E. Blandre, L. Chaput, S. Merabia, D. Lacroix, and K. Termentzidis, Phys. Rev. B 91, 115404 (2015).

[29] Y. Zhou and M. Hu, Nano Lett. 16, 6178 (2016).

[30] S. Plimpton, J. Comput. Phys. 117, 1 (1995).

[31] D. J. Evans and G. P. Morriss, Statistical Mechanics of Nonequilibrium Liquids, 2nd ed. (ANU E Press, Canberra, 2007).

[32] G. J. Ackland, MRS Adv. 1, 2857 (2016).

[33] A. Nichol and G. J. Ackland, Phys. Rev. B 93, 184101 (2016).

[34] F. H. Stillinger and T. A. Weber, Phys. Rev. B 31, 5262 (1985).

[35] R. Vink, G. Barkema, W. van der Weg, and N. Mousseau, J. Non-Cryst. Solids 282, 248 (2001).

[36] S. Munetoh, T. Motooka, K. Moriguchi, and A. Shintani, Comput. Mater. Sci. 39, 334 (2007).

[37] C. J. Glassbrenner and G. A. Slack, Phys. Rev. 134, A1058 (1964).

[38] P. Maycock, Solid-State Electron. 10, 161 (1967).

[39] V. Jean, S. Fumeron, K. Termentzidis, S. Tutashkonko, and D. Lacroix, J. Appl. Phys. 115, 024304 (2014).

[40] V. Jean, S. Fumeron, K. Termentzidis, X. Zianni, and D. Lacroix, Int. J. Heat Mass Transf. 86, 648 (2015).

[41] M. G. Holland, Phys. Rev. 134, A471 (1964).
[42] $\lambda_{\text {dominant }} \simeq h c / k_{B} T \simeq 1 \mathrm{~nm}$ for silicon at $T=300 \mathrm{~K}$ [43], $h$ is the Planck constant, $c$ the sound velocity in silicon, and $k_{B}$ the Boltzmann constant.

[43] R. Chen, A. I. Hochbaum, P. Murphy, J. Moore, P. Yang, and A. Majumdar, Phys. Rev. Lett. 101, 105501 (2008).

[44] M. Verdier, D. Lacroix, and K. Termentzidis, Int. J. Heat Mass Transf. 114, 550 (2017).

[45] H. Wada and T. Kamijoh, Jpn. J. Appl. Phys. 35, L648 (1996).

[46] M. Asheghi, M. N. Touzelbaev, K. E. Goodson, Y. K. Leung, and S. S. Wong, J. Heat Transfer 120, 30 (1998).

[47] P. B. Allen, J. L. Feldman, J. Fabian, and F. Wooten, Philos. Mag. B 79, 1715 (1999).

[48] Z. Liang, T. E. Wilson, and P. Keblinski, J. Appl. Phys. 121, 075303 (2017).

[49] A. J. Scholten and J. I. Dijkhuis, Phys. Rev. B 53, 3837 (1996).

[50] R. Markiewicz, J. Phys. Chem. Solids 58, 1179 (1997).

[51] Y. He, D. Donadio, J.-H. Lee, J. C. Grossman, and G. Galli, ACS Nano 5, 1839 (2011).

[52] E. Chávez-Ángel, J. S. Reparaz, J. Gomis-Bresco, M. R. Wagner, J. Cuffe, B. Graczykowski, A. Shchepetov, H. Jiang, M. Prunnila, J. Ahopelto, F. Alzina, and C. M. Sotomayor Torres, APL Mater. 2, 012113 (2014).

[53] Z. Wei, G. Wehmeyer, C. Dames, and Y. Chen, Nanoscale 8, 16612 (2016).

[54] S. W. Lovesey, Condensed Matter Physics: Dynamic Correlations, 2nd ed. (Benjamin Cummings, Menlo Park, CA, 1986).

[55] W. Humphrey, A. Dalke, and K. Schulten, J. Mol. Graphics 14, 33 (1996).

[56] A. Henry and C. Gang, J. Comput. Theor. Nanosci. 5, 141 (2016). 